\title{
Surveillance of duodenal adenomas in familial adeno- matous polyposis patients: medical objectives and technical requirements
}

Authors

Institution
Jean-Christophe Saurin, Mathieu Pioche, Jérôme Rivory

Edouard Herriot Hospital, Gastroenterology, Lyon

\section{Bibliography}

DOI http://dx.doi.org/

10.1055/s-0034-1393130

Published online: 8.10.2015

Endoscopy International Open

2015; 03: E456-E457

(c) Georg Thieme Verlag KG

Stuttgart · New York

E-ISSN 2196-9736

Corresponding author

Jean-Christophe Saurin

Edouard Herriot Hospital Gastroenterology unit

Pavillon $L 2^{\circ}$ et.

Hôpital E. Herriot

5 Pl d'Arsonval

69437 Lyon Cedex 03

France

Phone: +33472110369 jean-christophe.saurin@chulyon.fr
License terms

(이 (1) $\ominus \circledast$
Patients with classical familial adenomatous polyposis (FAP) present with hundreds of colorectal adenomas requiring (sub)total colectomy at around age 20 years, and frequently duodenal adenomas. Duodenal polyposis is a progressive disease. The burden of duodenal adenomas evolves more slowly as compared to colorectal adenomas, but increases with age to the highest stage of duodenal polyposis, stage IV according to the Spigelman's classification, with an expected cumulative frequency of $50 \%$ at age 70 years [1]. Although the surveillance recommendations are relatively clear and homogeneous in the different countries, with the first duodenal surveillance between 20 and 25 years, the modalities of treatment are much less well defined.

Duodenal adenomas either ampullary or periampullary present usually as flat, whitish, small lesions and slightly elevated as compared to the surrounding mucosa. There is a majority of small $(<5 \mathrm{~mm})$ lesions, and some larger lesions depending on the severity of the duodenal polyposis. Endoscopists have to focus on the detection and characterization of larger $(>1 \mathrm{~cm})$ lesions as these have been shown to contain foci of high-grade dysplasia in up to $50 \%$ of cases and have been associated with a risk of cancer development.[2, 3] The role of the endoscopist during duodenal surveillance is to 1 ) detect any existing cancer (complete evaluation of the duodenum and firsts jejunal loops); and 2i) evaluate the burden of duodenal adenomas in order to estimate the risk of advanced neoplastic evolution and the adapted surveillance interval. The only available criterion nowadays is the Spigelman's classification, which includes the number $(<10,10-20,>20)$, the size $(<5 \mathrm{~mm}, 5-10 \mathrm{~mm},>10 \mathrm{~mm})$ and the histology (low- or high-grade dysplasia) of duodenal adenomas. Optimal endoscopic examination of the duodenum, with lateral viewing and axial viewing at the same time, using a long endoscope to visualize the proximal jejunum and indigo-carmine to improve visualization of the mucosa, although relatively consensual for expert centers, has not really been validated through prospective studies. At least, indigo-carmine dye has been shown to increase the number of adenomas detected in two series and to increase the Spigelman's score. $[4,5]$

The question addressed in the paper by Pittayanon and colleagues [6] is that of the diagnostic value of new imaging methods (narrow band imaging and confocal microscopy) as regards the identification of duodenal adenomas. The main clinical question in this disease should be: What are the limitations of present surveillance modalities, what is the reference method of examination, and do we need new methods to improve surveillance? The main limitation of the usual surveillance of duodenal polyposis would be to overlook major neoplastic area that would evolve into cancer during the recommended surveillance interval ( $2-3$ years). Some old retrospective series suggest such a limitation, but these are mainly based on a largely obsolete methodology (axial viewing, old endoscopes, no sedation) [3]. In contrast, recent prospective series, even in patients with severe duodenal polyposis, do not suggest a high frequency of overlooked precancerous lesions [7]. Finally, the recent progress of usual endoscopes into high-definition endoscopy will probably replace, based on our experience, even indigo-carmine dye, given the excellent visualization of even tiny adenomas. And this evolution is still underway, given the new generation of endoscopes that are under development or research. Thus, there will probably be no clinical need for new technologies to identify more duodenal adenomas in FAP patients.

On the other hand, is it important to differentiate duodenal adenomas from other duodenal polyps, as proposed by Pittayanon and colleagues? There is a real concern regarding the duodenal bulb as some lesions can mimic duodenal adenomas in 
this area, including gastric metaplasia and Brunner glands. There is also a question regarding the identification of adenomatous tissue on the duodenal papilla, especially because biopsies, although with a very low frequency, can induce pancreatitis. In the latter case, however, it would be important to determine whether microscopic (macroscopically invisible) ampullary adenoma represent a danger for patients even on a long-term basis, given the very slow evolution of duodenal adenomas [8]. In our experience, we usually leave in place small, flat, visible ampullary adenomas for years. Regarding the numerous small duodenal adenomas observed throughout the proximal and distal duodenum of patients with FAP, there is no recommendation to biopsy and thus confirm the diagnosis of adenomas, as there is almost no differential diagnosis in this area. Practically, we never take numerous biopsies of these lesions, but simply count the number of adenomas to get a satisfying evaluation of the Spigelman's score. For these reasons, identifying exactly the nature of small whitish lesions in the proximal and distal duodenum is probably of low clinical relevance. Moreover, if this was of any clinical relevance, any new diagnostic method should be compared with simple, white-light imaging, and probably to the reference method, i.e indigo carmine dye.

Finally, what is important for patients with FAP regarding duodenal examination? Three measures: 1 ) beginning relatively early surveillance, around age 20 years, as we observe severe polyposis in a low number of young patients; 2 ) following recommended modalities of examination, including anesthesia, lateral and axial viewing, examination of the proximal jejunum to identify all possible areas of advanced neoplasia; and 3 ) being very cautious with large $(>1 \mathrm{~cm})$ adenomas, which should be removed because they represent a significant risk of high-grade dysplasia or cancer. We should keep in mind that this evaluation needs to be done at regular intervals, is time consuming, and should remain relatively simple so that gastroenterologists can keep on doing an excellent and regular evaluation of this significant risk of duodeno-jejunal cancer in patients with FAP.

\section{Competing interests: None}

\section{References}

1 Saurin JC, Gutknecht C, Napoleon B et al. Surveillance of duodenal adenomas in familial adenomatous polyposis reveals high cumulative risk of advanced disease. J Clin Oncol 2004; 22: 493 - 498

2 Saurin JC, Ligneau B, Ponchon T et al. The influence of mutation site and age on the severity of duodenal polyposis in patients with familial adenomatous polyposis. Gastrointest Endosc 2002; 55: 342 - 347

3 Groves CJ, Saunders BP, Spigelman AD et al. Duodenal cancer in patients with familial adenomatous polyposis (FAP): results of a 10 year prospective study. Gut 2002; 50: 636-641

4 Dekker E, Boparai KS, Poley JW et al. High resolution endoscopy and the additional value of chromoendoscopy in the evaluation of duodenal adenomatosis in patients with familial adenomatous polyposis. Endoscopy 2009; 41: 666-669

5 Picasso $M$, Filiberti $R$, Blanchi $S$ et al. The role of chromoendoscopy in the surveillance of the duodenum of patients with familial adenomatous polyposis. Dig Dis Sci 2007; 52: $1906-1909$

6 Pittayanon $R$, Reknimitr $R$, Imraporn $B$ et al. Diagnostic values of dual focus narrow band imaging and probe-based confocal laser endomicroscopy in FAP-related duodenal adenoma. Endosc Int Open DOI 10.1055/s-0034-1392235

7 Moussata D, Napoleon B, Lepilliez V et al. Endoscopic treatment of severe duodenal polyposis as an alternative to surgery for patients with familial adenomatous polyposis. Gastrointest Endosc 2014: pii: 5107 (14)01237-1. pii: DOI 10.1016/j.gie.2014.03.012

8 Iida $M$, Yao T, Itoh $\mathrm{H}$ et al. Natural history of duodenal lesions in Japanese patients with familial adenomatosis coli (Gardner's syndrome). Gastroenterology 1989; 96: $1301-1306$ 